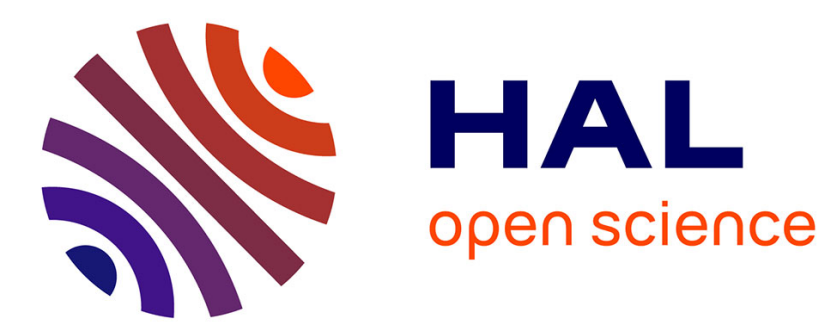

\title{
LE PROCÉDE KRAUSE POUR LA FABRICATION DE LA POUDRE DE LAIT
}

\author{
Leon Panchaud
}

\section{To cite this version:}

Leon Panchaud. LE PROCÉDE KRAUSE POUR LA FABRICATION DE LA POUDRE DE LAIT. Le Lait, 1924, 4 (35), pp.369-381. hal-00894768

\section{HAL Id: hal-00894768 \\ https://hal.science/hal-00894768}

Submitted on 1 Jan 1924

HAL is a multi-disciplinary open access archive for the deposit and dissemination of scientific research documents, whether they are published or not. The documents may come from teaching and research institutions in France or abroad, or from public or private research centers.
L'archive ouverte pluridisciplinaire HAL, est destinée au dépôt et à la diffusion de documents scientifiques de niveau recherche, publiés ou non, émanant des établissements d'enseignement et de recherche français ou étrangers, des laboratoires publics ou privés. 
mes expériences par la chaleur, elle se manifeste de mème mais plus lentement au contact de l'air ambiant en fonction de la température et de l'humidité : c'est le cas de la pratique.

Il me paraît intéressant d'illustrer cette explication par un exemple frappant. Supposons en effet que l'on mélange intimement dans un flacon sec, du bicarbonate de soude et de l'acide tartrique, l'un et l'autre finement pulvérisés et très secs. Malgré la vive affinité des produits en contact, aucun phénomène ne se produira tant qu'on les conservera à l'abri de l'humidité. Faisons tomber une goutte d'eau en un point quelconque du mélange: immédiatenent l'acide tartrique agira activement sur le bicarbonate à ce seul point. Ouvrons maintenant le flacon et laissons-le débouché, exposé à l'air. L'humidité de l'air ambiant se fixera sur nos produits et favorisera leur contact, progressivement, et beaucoup plus lentement que dans le cas de la goutte d'eau, l'acide tartrique réagira sur le bicarbonate qui sera à la longue totalement décomposé.

Le taux d'humidité fixée ou non éliminée, joue done un rôle très important sur la persistance de la solubilité des poudres de lait. La poudre A que j'ai utilisée contenait $6.8 \%$ d'eau, condition favorable pour provoqner peu à peu son insolubilité, comme je l'ai constaté, tandis que la poudre $\mathrm{B}$ contenait seulement $1,6 \%$ d'humidité, ce qui explique sa longue conservation. Comme dans l'exemple ci-dessus, et ainsi que je l'ai démontré dans mes expériences, l'humidité acquise ou non suffisamment éliminée au moment de la fabrication, favorise, ou, mieux encore, provoque l'action insolubilisante de l'acide lactique sur la caséine de la poudre de laịt.

Il est aisé de tirer une conclusion pratique de cet exposé. Le fabricant de poudre de lait, qui poursuivrale but d'obtenir une poudre susceptible de conserver toute sa solubilité,devra, lors de la fabrication,réduire le taux d'humidité à environ $3 \%$ et maintenir ce taux constant dans le produit livré au commerce, par un emballage soigné, afin d'éviter toute humidification au contact de l'air.

\title{
LE PROGEDE IRRAUSE POUR LA FABRICATION DE LA POUDRE DE LAIT,
}

\author{
par LÉon PANCHAUD,
}

Docteur ès Sciences, attaché au Laboratoire Cantonal d'Analyses de Genève (Suisse).

Depuis quelques années (1918), les périodiques de langue allemande consacrent de nombreux articles aux différents produits (poudre de lait, poudre de sang, poudre d'œufs, etc...) obtenus par 
un procédé d'éváporation dù à l'ingénieur Krause, de Munich. " Le Lait » a déjà rendu compte de quelques articles publiés en langue allemande sur la poudre de lait Krause et ses applications à l'alimentation infantile.

Il m'a semblé intéressant, étant donné l'importance et la nouveauté du sujet, de résumer pour les lecteurs du "Lait " les publications relatives à ce procédé, afin d'en montrer les possibilités d'application et de donner une idée de l'appareillage nécessaire à sa réalisation.

Le but que l'on se propose dans la fabrication de la poudre de lait n'est pas seulement l'obtention d'un produit de volume restreint, de longue conservation et de transport commode, mais surtout d'un produit qui, par restitution de l'eau évaporée donne un liquide ayant tous les caractères organoleptiques, physico-chimiques, biologiques et nutritifs du lait originel. La poudre de lait idéale doit se dissoudre rapidement dans l'eau sans laisser de résidu insoluble, le liquide obtenu doit faire crême, coaguler sous l'influence de la présure tout comme le lait frais et ne pas avoir le goùt de " cuit ».

Pour dessécher un liquide aussi sensible que le lait, où de grandes quantités doivent pouvoir être transformées rapidement en évitant des températures élevées de nature à compromettre sa nature physico-chimique, il faut chercher à augmenter la surface du lait :

$\left.1^{\circ}\right)$ en répandant le lait en nappe mince sur des surfaces chaudes (proeédé Just);

$2^{\circ}$ ) en faisant agir un courant d'air chaud ou de vapeur sur le lait pulvérisé :

a) soit par passage sous pression dans une série de tubes capillaires (procédé Trufood, 1906, spray-process) ;

b) soit par passage sur un disque animé d'une très grande vitesse de rotation qui le centrifuge en un brouillard impalpable (procédé Meister D. K. P. 2כ้4.992, procédé Krause D. R. P, 297.388).

Mais la chaleur accélère toute réaction, elle tend à troubler l'équilibre physico-chimique des colloïdes, à les faire passer d'un état stabile favorable à un état labile défavorable, que l'on veut justement éviter c'est-à-dire que, sous l'action de la chaleur, la forme « sol » passe à la forme " gel » ou à un état intermédiaire irréversibles, de sorte que le produit desséché ne se dissout plus ou imparfaitement dans le solvant primitif. Ces réactions indésirables s'effectuent en peu de temps, il faut donc, si on veut les éviter, amener, dans un laps de temps plus court encore, l'état liquide, sans élever sa température, à un état suffisamment " anhydre " pour que ces réactions n'aient pas le temps de s'effectuer. A l'état sec, les colloïdes les plus sensibles peuvent alors supporter l'action des températures élevées $\left(120-130^{\circ}\right)$ sans que leurs propriétés primitives soient modifiées. 
Ces conditions se trouvent réalisées dans le procédé Krause ; parpar suite d'une pulvérisation poussée à l'extrême limite, le lait est instantanément desséché.

On sait que la poudre obtenue selon le procédé Just ne se dissont pas toujours intégralement dans l'eau, la température élevée à laquelle la dessiccation a lieu provoque une dénaturation des albuminoïdes, de sorte que le lait obtenu par dissolution de la poudre dans l'eau ne présente plus les caraetères biologiques du lait frais. La poudre de lait Just ne se conserve pas toujours d'une façon satisfaisante.

La poudre de Iait obtenue selon le procédé Trufood se dissout très rapidement et complètement dans l'eau, à condition de n'être pas trop vieille; le liquide obtenu possède tous les caractères physicochimiques et biologiques du lait cru. Ce procédé ne serait pas. rémunérateur ;une usine installée à Ragnitz, en Prusse, aurait dâ cesser son exploitation après 2 ans. Les tubes pulvérisateurs s'obstruaient facilement, ce qui provoquait des arrêts dans la fabrication; le rendement était de $10-15 \%$ inférieur à celui du procédé Just par suite de dispositifs de filtration insuffisants. La poudre Trufood « Milchfix " se conservait mal, donnait un lait à odeur suiffeuse laissant passablement de résidu insoluble. Ces altérations étaient dues à la finesse de la poudre, qui favorisait une oxydation rapide du beurre et une saturation de la chaux liée à la caséine par les acides gras formés, d'où insolubilité relative de la caséine.

Dans le procédé Krause, la pulvérisation est obtenue par l'intermédiaire d'un disque placé à l'intérieur d'une tour. Ce disque tourne, suivant son diamètre, à une vitesse de 5.00024 .000 tours par minute. Le lait arrive au centre du disque par un tuyau d'alimentation et, par le jeu complexe des forces centrifuge, de cohésion et d'adhésion, il est projeté, avec une vitesse considérable, sous forme d'un nuage d'une extrême finesse. Un ventilateur aspire de l'air châud qui traverse le nuage, lequel est desséché avant même qu'il ait atteint les parois de la tour, e'est-à-dire en une traction de seconde. Les particules desséchées tombent de leur propre poids sur le sol de la tour, comme une " crachée de neige ». La mesure microseopique du diamètre des poudres obtenues, dans l'appareil de Krause, par dessiccation de solutions de concentration connue, montre que le liquide est pulvérisé en particules dont le diamètre varie entre 10-50 $\mu$. Un litre de solution occuperait, dans ees conditions, une superficie de $300 \mathrm{~m}^{2}$.

Le disque, au début, tourne avec une vitesse tangentielle de $160 \mathrm{~m}$. par seconde. Les gouttelettes de lait quittent le disque avec cette vitesse, à peine modifiée par le travail de pulvérisation. Dans les cas les plus défavorables, la résistance desl'air la diminue de moitié, les 
particules ont encore une vitesse de $80 \mathrm{~m}$. par seconde. Le diamètre de la tour étant de 2-5 $\mathrm{m}$, , une gouttelette de liquide quittant le disque mettra $1 / 80-1 / 28^{\circ}$ de seconde, pour arriver contre les parois de la tour, et comme elle doit être desséchée avant d'y parvenir, on voit que la dessiecation se fait en une fraction infime de seconde, $1 / 40$ e en moyenne.

D'autre part, la vitesse d'évaporation d'un liquide, à température eonstante dépend de deux facteurs : grandeur des surfaces de contact -entre l'air et le liquide et rapidité de remplacement des couches d'air enrichies en vapeur d'eau, en eontact avec le liquide, par d'autres couches d'air contenant moins d'humidité. Ces deux facteurs atteignent dans le procédé Krause, une valeur extraordinairement élevée, le disque communique au liquide une énergie cinétique considérable, la surface du liquide augmente dans des proportions énormes et les particules acquièrent une vitesse considérable, ec qui favorise une évaporation intense. Dans ces conditions, l'évaporation du liquide se fait si rapidement que la dessiccation a lieu adiabatiquement. La majeure partie de l'eau des particules s'est évaporée sans que la chaleur nécessaire ait été empruntée au courant d'air chaud, elle a été prise en les particules même, de sorte que leur température s'abaisse. Tant que les particules sont encore humides, elles ne peuvent être exposées à une températare supérieure à celle qui correspond à la valeur calorifique de l'air quiles entoure La valeur calorifique d'un kilo d'air sec, porté à $150^{\circ}$ (température à laquelle il entre dans l'appareil de KrAuse) est de 35,6 calories. Lorsque cet air est complètement saturé de rapeur d'eau, sa température s'est abaissée à 3 ă$^{\circ}$. L'air chaud employé pour la dessiccation ayant généralement une humidité relative de $50 \%$ à $20^{\circ}$, sa température s'abaisse en réalité à 4. $4^{\circ}$ Les particules, tant qu'elles ne sont pas complètement desséchées, ne sont pas exposées à une température supérieure à $41^{\circ}$. Le brouillard presque desséché arrive en contact avec le courant d'air chaud qui se sature en humidité ; la force du courant est calculée de telle manière que la majeure partie des particules desséchées puissent tomber de leur propre poids sur le sol, et se dessécher complètement au contact d'air de moins en moins riche en vapeur d'eau.

Une installation Krause comprendra les organes essentiels suivants :

Un système de filtration de l'air frais $A$, dans lequel lair se dépouille de ses poussières avant de pénétrer dans le chauffeur $B$, où sa température est portée de $120-130^{\circ}$;

Une tour $\mathrm{C}$ dans laquelle se font la pulvérisation et la dessiccation;

Un système de tuyaux-filırants $D$, qui retiennent les fines porlions de poudres entrainees avec le courant d'air chaud; 
Un turbo-ventilateur E.-F., pour le réglage du courant d'air et l'expulsion de l'air à sa sortie des filtres;

L'organe essentiel, la " tour » de Krause, est un cylindre tronc-conique en béton, revêtu intérieurement de briques émaillées. A l'intérieur de la tour se trouve la tourelle portant le disque pulvérisateur $\mathrm{H}$, dont le diamètre est de $40-50 \mathrm{~cm}$., mis en mouvement par une turbine à vapeur $\mathrm{T}$ disposée sous la coupole de la tourelle de telle façon que la vapeur ne pénètre pas dans l'intérieur de la tour de dessiccation.

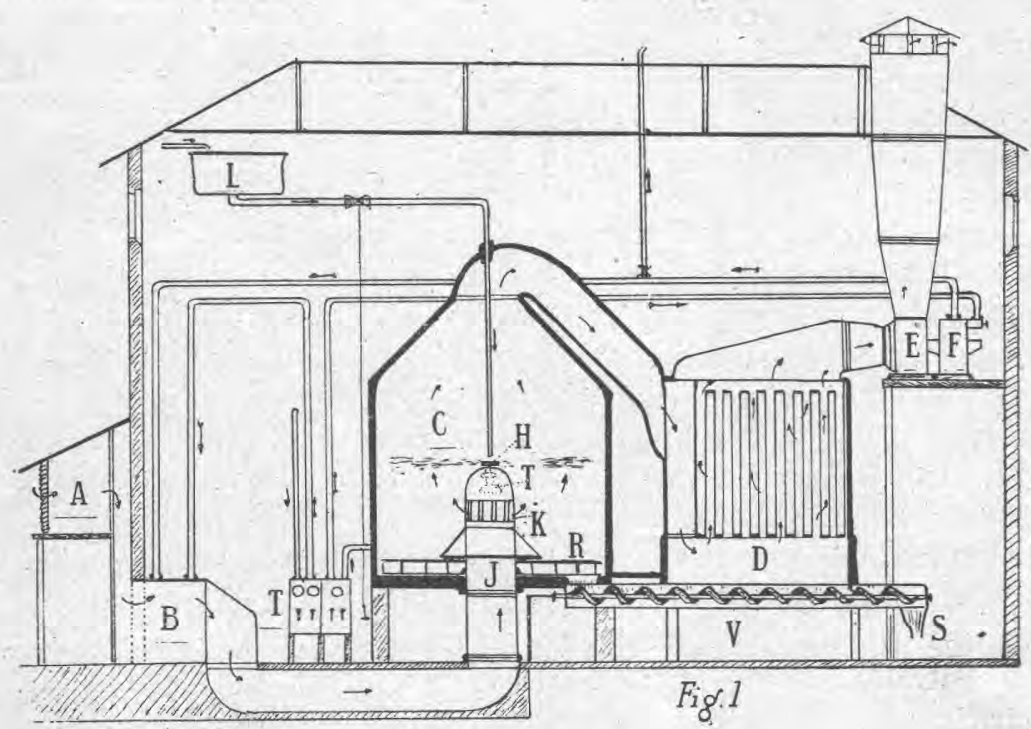

A Filtration de l'air frais.

B Chambre de chauffage de l'air filtré.

C Tour de Kratse.

$J$ Tourelle pourvue des soupapes $K$ et renfermant la turbine $T$ mettant en mouvement le disqne pulvérisateur $\mathbf{H}$.

R Raclette conduisant la poudre de lait sur la vis sans fin V.

D Dispositif a tuyaux-filtres $n$.

F Turboventilateur pour l'aspiration et l'expiration de l'air.

S Sortie de la poudre de lait (appareils d'ensachage).

T Tableau de distribution pourvu des appareils pour règler la force du courant d'air et sa température, la vitesse de rotation du disque, etc...

Par le bas de la tour, en J, pénètre l'air chaud; un jeu de soupapes K permet de régler à volonté l'admission de l'air. Le lait, arrivant d'un réservoir d'alimentation I par un tuyau débouchant au milieu du disque est entraîné contre le bord du disque et centrifugé en un brouillard impalpable, qui est instantanément dess śché. Une partie de 
la poudre tombe de son propre poids au fond de la tour, un rateau $\mathbf{R}$. la conduit sur une vis sans fin V. Une certaine proportion de la poudre, la plus fine, est entrainée avec le courant d'air chaud; elle est retenue sur les tuyaux-filtrants $\mathrm{D}$, un dispositif mécanique les secoue automatiquement à intervalles réguliers et fait tomber la poudre sur la vis sans fin et de lì dans les appareils d'ensachage. en $\mathbf{S}$.

On peut pénétrer dans la tour de dessiccation par une porte pourvue d'une fenetre d'observation.

La marelıe de la fabrication se règle depuis une table centrale $\mathrm{T}$ qui comporte tous les appareils pour le réglage, soit de l'admission de l'air, soil du lait, etc...

Les appareils sont construits généralement pour évaporer 1.000 . litres d'eau par heure; mais, leur rendement étant généralement supérieur au rendement garanti, on peut très facilement évaporer avec un appareil de 1.000 litres, 1.500 litres de lait par heure.

D'autres apparcils sont calculés pour évaporer 500 litres d'eau par heure (1abrieation de la poudre de sang, d'œufs, ete..). Il existe même des appareils pouvant évaporer 5 litres d'eau par heure (expériences de laboratoire, dessiecation des sérums...) qui sont mus alors uniquement au moyen de l'électricité.

La construction des appareils ne se fait pas suivant un sehéma invariable, elle est, en quelque sorte, fonction du liquide à dessécher. C'est ainsi que le liquide pent arriver soit par la face supérieure, soit par la face inférieure du disque; la turbine portantle disque peut être fixée soit au sol, soit au sommet de la tour (dans l'évaporation du latex, par exemple). L'arrivée et la direction du courant d'air peuvent être centrales ou tangentielles, de bas en haut et vice-persa. Au lieu d'air chauffé à la vapeur, on peut, lorsqu'il ne s'agit pas de denrées alimentaires, employer des gaz résiduaires chauds.

Pour évaporer 1.000 litres d'eau, il faut disposer de 50.000 mètres eubes d'air à $130^{\circ}$.

Un correspondant de la Schweizerisches Zentralblatt für Milchwirtschaft (27 janvier 1921) donne une description assez intéressante de l'usine installée à Sulgen (Suisse) pour la fabrication de poudre de lait selon Kriuse. Le battiment principal, d'aspect imposant, renfermant les apparcils de dessiccation, est relié par une ligne spéciale avec li station même de Sulgen. Un autre bâtiment, relié au précédent par un tunnel souterrain, comprend les appareils de chauffe, chaudières, ctc... Il comporte 2 chaudières de $200 \mathrm{~m}^{2}$ desurface de chauffe chacune, $\epsilon$ i pouvant produire chacune normalement 3.600 kilos de vapeur quantité pouvant être portée à 4.400 kilos en forçant les feux. L'eau de condensation dont la température est relativement élevée est pompée directement dans les chaudières, de: 
telle façon que la quantité d'eau fraîche de réalimentation n'est que de $10 \%$ environ.II y a done forte économie de combustible et diminu. tion des incrustations de chaudières. Dans le battiment principal, d'une hauteur imposante, se trouvent outre la " tour » et les dispositifs de filtration, les appareils de filtration du lait, de condensation du lait ou du petit lait dans le vide, le réchauffeur d'air et les machines à froid pour la réfrigération du lait à son arrivée à l'usine. La tour de dessiccation a un diamètre interne de $\breve{~ m e ̀ t r e s, ~ s a ~ h a u t e u r ~}$ est de 8 mètres. Le réchauffeur d'air peut porter par heure $50.000 \mathrm{~m}^{3}$ d'air de la température ordinaire à $130^{\circ}$. Le turboventilateur qui assure l'aspiration et l'expulsion de l'air chaud peut débiter $60.000 \mathrm{~m}^{3}$ d'air par heure. L'usine évapore 1.500 litres de lait par heure; en combinant la condensation avec la dessiccation, on peut arriver à dessécher 3.000 litres de lait par heure (les appareils de condensation évaporant 1.500 litres d'eau par heure). L'usine eomprend encore le local de réfrigération du lait, qui comporte 14 baes de réfrigération de 3.000 litres de contenance environ.

On ne doit évaporer qu'un lait de fraîcheur irréprochable, e'est-àdire résistant à l'essai à- l'alcool ou à l'alcool-alizarine (réactif de Morres). Les laits aigris, acidulés, "piqués 》 ne peuvent servir à la fabrication de la poudre de lait.

Le procédé Krause permet de dessécher toutes les substances que l'on peut mettre en solution ou en suspension homogène (solutions colloïdales, boues, produits fondus, etc. .). Il est économique à condition que la teneur en eau du produit à dessécher soit inférieure à $90 \%$; lorsqu'elle est supérieure à $90 \%$, il taut recourir à une condensation préalable dans le vide.

On obtient toujours une poudre très fine, sans modifications appréciables de la nature physico-chimique. Les poudres Krause, par redissolution dans l'eau, sont la reconstitution exacte du produit original. Le complexe colloïdal (lait, cufs, sang, etc...) n'est pas sensiblement modifié, les ferments ont conservé pratiquement leur entière activité. En restituant à la poudre de lait l'eau évaporée, on obtient un lait qui donneles réactions de Storch, de Rothenfusser(oxydases), et qui décolore le réactil de Schardinger (bleu de méthylène formolé) en 13 minutes $1 / 2$ alors que le lait originel le décolore en 11 minutes. La eatalase du sang n'est pas détruite dans la poudre de sang Krause, on peut espérer que la vitamine $\mathrm{C}$ antiscorbutique, de même que les

- vitamines A et B, si facilement oxydables, restent intactes dans les poudres Krause.

La teneur en eau des poudres Krause est comprise entre 2-5 \% . $\mathrm{Vu}$ leur extrême finesse, leur couleur très claire, elles doivent être conservées à l'abri de la lumière et autant que possible dans un 
endroit frais et sec. Dans ces conditions, la poudre de lait se conserve pendant 6 mois sans altération appréciable. Elle prend à la longue (poudre de lait entier) un goût de rance ou plutôt de suiffeux,par suite -d'une lente décomposition de la matière grasse. La poudre de lait écrémé se conserve plus longtemps.

Il suffit pour avoir un lait entier de dissoudre $130 \mathrm{gr}$. de poudre de lait entier dans un litre d'eau froide ou tiède. En délayant peu à peu la poudıe avec l'eau, la dissolution se fait rapidement et complètement.

J'ai eu entre les mains une poudre de lait entier KraUse, elle contenait $29 \%$ de beurre, de sorte que le lait obtenu à partir de $130 \mathrm{gr}$. de poudre et 1 litre d'eau, renfermait $3,8 \%$ de beurre. Cette poudre représentaịt done bien le produit de la dessiccation d'un lait entier. La saveur du produit obtenu par dissolution dans l'eau est celle du lait frais; le liquide fait crème absolument comme le lait frais Je l'ai conservée pendant plus de 3 mois dans un poudrier a l'inté rieur d'un tiroir, près d'un radiateur, à l'abri de la lumière, à la température du laboratoire ; au bout de ce temps, elle donne encore un lait irréprochable comme goût, aspect et acidité, se comportant normalement à l'essai à l'alcool-alizarine; la solubilité de la poudre ne semble pas avoir souffert le moins du monde.

Pendant la guerre, la poudre de lait Krause fut réservée aux troupes ; la guerre finie elle fut expérimentée avec suecès pour l'alimentation de la population eivile et dans les hôpitaux, à Berlin et Francfort par Czerny, Neuland et Peiper et von Noorden.

En ce qui concerne l'alimentation infantile, les résultats des expériences ont été des plus satisfaisants; les enfants ont à leur disposition un lait contenant peu de germes, en tout cas moins de germes que le lait frais du commerce. Ce facteur a une importance considé. rable dans l'alimentation infantile; beaucoup de troubles nutritif peuvent être évités ou guéris par l'administration de la poudre de lait. Pour Czerny, l'introduction de la poudre de lait entier dans l'alimentation infantile constitue un progrès énorme. Les vitamines sont certainement intactes dans la poudre de lait KrAUSE ; mais par mesure préventive, on administre aux enfants nourris à la poudre de lait des sucs de fruits riches en vitamines antiscorbutiques (oranges, tomates) 2-3 fois par semaine.

Pour beaucoup, la question du ravitaillement en lait des grands centres apparait sous un jour nouveau ; par le procédé KrAUse, on. pourra accumuler ainsi la surproduction des grands centres laitiers pour les périodes de disette; la question du transport du lait frais à distance, si délicate, serait ainsi résolue.

Le ravitaillement en lait de la ville de Francfort-sur-le-Main est 
difficile, cette ville étant entourée d'un région peu propice à l'élevage du bétail. Le lait frais vient de contrées éloignées et arrive souvent impropre à la consommation pendant la saison chaude. La municipalité fait dessécher le lait dans l'usine de Kappeln, distante de plus de 700 kilomètres, en plein centre raitier du Schleswig. Dans cette usine, deux appareils Krause, type 1000 litres d'eau à l'heure, évaporent le lait qui est expédié sous forme de poudre à Francfort. Sous contrôle municipal, cette poudre est dissoute dans l'eau et, le lait ainsi obtenu, est vendu à la population qui ne peut pas le différencier du meilleur lait frais.

Les avantages teclnniques du procédé Krause sont:

1. Obtention dans tous les eas d'un produit final en poudre fine et légère, ce qui rend inutiles les opérations de mouture, tamisage, etc., même dans le cas de solutions colloïdales ou de suspensions que les procédés ordinaires ne permettent d'obtenir qu'en croûtes ou masses compactes qui doivent être moulues tamisées avant dêtre livrẻes à la consommation.

2. Obtention directe du produit final sans produits intermédiaires ; la fabrication peut être interrompue en tout instant, sans crainte d'avaries de matériel.

Ces avantages permettent de résoudre des problèmes jusqu'à ce jour insolubles, puisqu'ils permettent de dessécher tous les produits sensibles à l'action de la chaleur en leur conservant leur caractère original (œufs, sang, colle, sucs de fruits, solutions de savon, suc de levure de Buchner, sérums thérapeutiques, légumes, etc.).

Wassermann a expérimenté des sérums desséchés selon Krause (sérum anticholérique et antithyphique obtenus en poudre blanche), sue de levure (obtenu en poudre légèrement jaunàtre). Les propriétés biologiques-immunisantes et fermentatives de ces produits n'avaient subi pratiquement aucune altération.

Le procédé Kn $\Lambda$ USE permet la dessiccation, par pulvérisation simultanée, de plusieurs solutions ou suspensions, il en résulte des mélanges d'une remarquable homogénité, ce qui évite les opérations souvent compliquées du mélange. Exemple : la fabrication de poudre au nitrate d'ammonium, à partir d'une solution de nitrate d'ammonium et d'une suspension de poudre de charbon, fabrication qui fut entreprise en grand pendant la guerre.

On peut aussi évaporer plusieurs corps dissous en une même solution. Lors de la concentration de pareilles solutions, il se produit généralement des précipitations, les corps les moins solubles précipitant les premiers, le produit desséché n'a plus aucune homogénéité. Ces solutions évaporées dans la tour de Krause donnent toujours comme produit final des poudres dont toutes les particules contiennent exactement les mêmes proportions de composants. 
C'est ainsi qu'on dessèche des extraits de drogues avec les substances servant à leur enrobage ou leur empastillage; les poudres obtenues, vu leur grande homogénéité sont d'une action thérapeutique constante (poudres de sucs de fruits avec sucre, extrait de thé ou de café avee sucre ou sucre et lait, etc...).

Les solutions de chlorure de cérium sont très sensibles à l'action de la chaleur, elles ne peuvent pas être évaporées sans qu'il se forme de l'oxychlorure de cérium, qui gêne dans l'électrolyse en milieu fondu. Pour éviter cette hydıolyse, on ajoutait aux solutions à évaporer, du chlorure de calcium ou d'ammonium, ce qui dans l'électrolyse nécessite une dépense supplémentaire de courant. Dans l'appareil de KrAUSE, on peut, sans aucune crainte de dégradation, évaporer la solution aqueuse de chlorure de cérium; la poudre obtenue ne renferme pas d'oxychlorure de cérium.

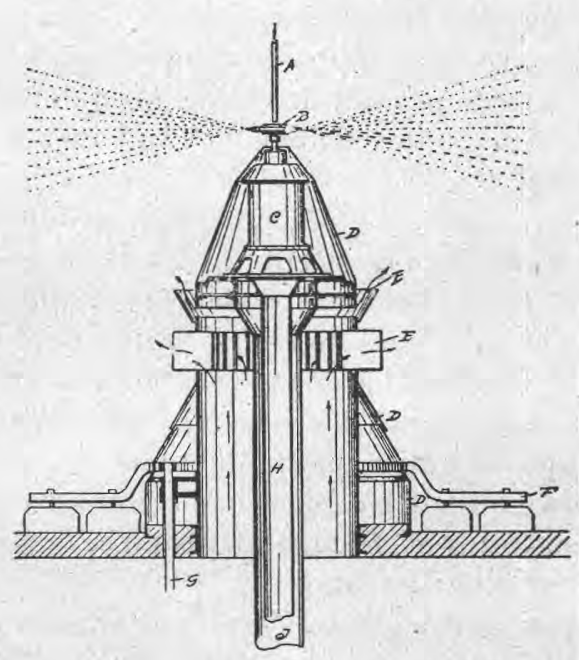

Appareillage pour la pulvérisation.

A Arrivée du liquide.

B Disque pulvérisateur.

C Moteur ou turbine à vapeur faisant tourner le disque

D Manteau protecteur.

E Sortie do l'air chaud.

F Bras du râteau.

G Dispositif d'eotraînement du râteau.

H Tuyau protecteur pour les cables, l'alimentation 'en huile et l'expulsion de l'air froid.

I Amenée d'air froid pour le refroidissement du moteur.

La préparation de digitale la plus active est l'extrait aqueux à froid, qui contient la digitaline et la digitaléine. La digitaline précipite 
à chaud, la digitaléine précipite à la longue même à la température ordinaire. L'extrait frais peut être évaporé au Krause en une poudre fine contenant les deux alcaloïdes parfaitement solubles dans l'eau, ce qui permet de préparer au moment même de l'emploi une solution d'action définie et toujours égale à elle-même, ce qui est important pour le médecin.

On peut également obtenir la caséine en poudre très fine d'une haute valeur plastique, des poudres d'oenfs entiers ou de jaune ou de blane séparément, sécher les bouillons de colle ou de gélatine, et ce, avec une grande économie de temps, d'espace et de personnel ; obtenir des poudres de savon (il s'agit alors plus d'un refroidissement rapide que d'une évaporation d'eau); certains appareils Krause sèchent 15 tonnes de savon par heure.

Le procédé Krause rend de grands services dans la fabrication du caoutchouc par évaporation directe du latex, additionné ou non des substances destinées à sa vulcanisation, à sa coloration ou à sa charge. 'On obtient une poudre contenant tous les éléments ajoutés également répartis. On peut aussi dessécher les solutions des corps très oxydables; l'évaporation de la solution d'indigo blane donne une poudre blanche, sans trace appréciable d'oxydation.

On peut même dessécher des légumes préalablement cuits, hâchés finement et passés à travers un tamis. La pâte obtenue est mise en suspension dans l'eau de cuisson et pulvérisée sur le disque. On obtient ainsi des poudres très claires et très légères, très homogènes et très digestibles, pouvant être administrées aux malades et même aux nourrissons (poudre de Friedenthal). Le prix de revient de telles poudres est malheureusement trop élevé pour que leur emploi se soit généralisé.

Pendant la guerre,en Allemagne, le proeédé Krause a servi à la préparation de poudre de sang pour les besoins de lá population civile. Le sang est un aliment de grande valeur puisqu'il contient environ $20 \%$ de matières solides (hémoglobine, albumine, cholestérine, lécithine et matières minérales). Le sang a donc une valeur alimentaire au moins égale à celle de la viande, valeur généralement inutilisée pour l'alimentation humaine.

On évaluait en Allemagne, avant la guerre,à 83.000 tonnes le poids du sang des animaux abattus annuellement.

En 1915, la erise alimentaire devenant très aiguë en Allemagne, on songea à dessécher en grand le sang. A Berlin, un appareil Krause (type 5̈00 litres à l'heure) desséchait le sang récolté dans les différents abattoirs.

Le sang était défibriné avant d'être desséché.

Le diamètre des grains de la poudre de sang était en moyenne de 
$50 \mu$ (maximum $116 \mu)$ dans la tour de dessiccation et $20 ّ \mu$ en moyenne (maximum $82 \mu$ ) pour la poudre récoltée sur les tuỹaux filtre.

Si le sang est desséché longtemps après l'abattage, le diamètre des. grains augmente légèrement [ ].

La couleur de la poudre de sang est légèrement plus foncée quecelle du sang trais.

Si à 1 partie de poudre de sang on ajoute par petites portions 4 parties d'eau, on obtient un liquide analogue au sang et qui coagulevers $70^{\circ}$. La poudre de sang, emballée dans de simples sacs en papier se conserve pendant 6 mois au moins, elle ne contient pas plus de germes que le beurre ou la margarine, par exemple. Le nombre des. germes diminue si la poudre contient du sel II est évident qne l'on ne peut et ne doit utiliser que le sang d'animaux sains pour la préparation de poudre de sang; la récolte du sang doit être faite avec précautions pour éviter les chances d'infection.

On peut aussi dessécher le sang non défibriné, d'où économie et. augmentation de la valeur alimentaire du produit. Le sang, s'il est coagulé passe avant d'arriver sur le disque pulvérislateur dans une machine à hâcher ou à homogénéiser. On peut aussi dessécher séparément le sérum sanguin, dont la poudre constitue un excellent succédané des oufs.

La poudre de sang sert à la préparation de boudins, de soupes.

A Munich, pendant la guerre, on vendait pour 25 pfennigs, 2 sachets. de $35 \mathrm{gr}$. contenant un mélange à base de poudre de sang $(2 \mathrm{kgr}$. poudre de sang, 5 kgr. farine, $259 \mathrm{gr}$. poudre d'oignons, $100 \mathrm{gr}$. marjolaine, 2ŏ gr. poivre et $10 \mathrm{gr}$. de paprika [ ]. Il suffisait de délayer le contenu d'un paquet avec un litre d'eau pour obtenir après cuisson une soupe des plus appétissantes, parait-il. La poudre de sang peut servir à l'affouragement des pores et constitue un fourrage concentré que les animaux supportent très bien.

Lorsque l'on évapore de l'eau dans un appareil chauffé avec de la vapeur d'eau, si l'appareil travaille sans aucune perte, il faut, théoriquement, employer une quantité de vapeur d'eau égale à la quantité d'eau évaporée. En pratique, par suite de pertes inévitables, la consommation de vapeur est supérieure de $20-30 \%$ à la quantité théorique.

Dans l'appareil de Krause, la chaleur de la vapeur n'est pas transmise directement à la substance à dessécher (comme dans les procédés d'évaporation par contact (Just...), mais par l'intermédiaire de l'air. Il n'y aurait rien de changé aux conditions théoriques, si l'air servant à la dessiccation était refroidi (par saturation en vapeur d'eau) à la température à laquelle il a été aspiré comme air frais. Celà est irréa-. lisable pratiquement, puisque la tension de la vapeur d'eau de l'air 
Figure I.

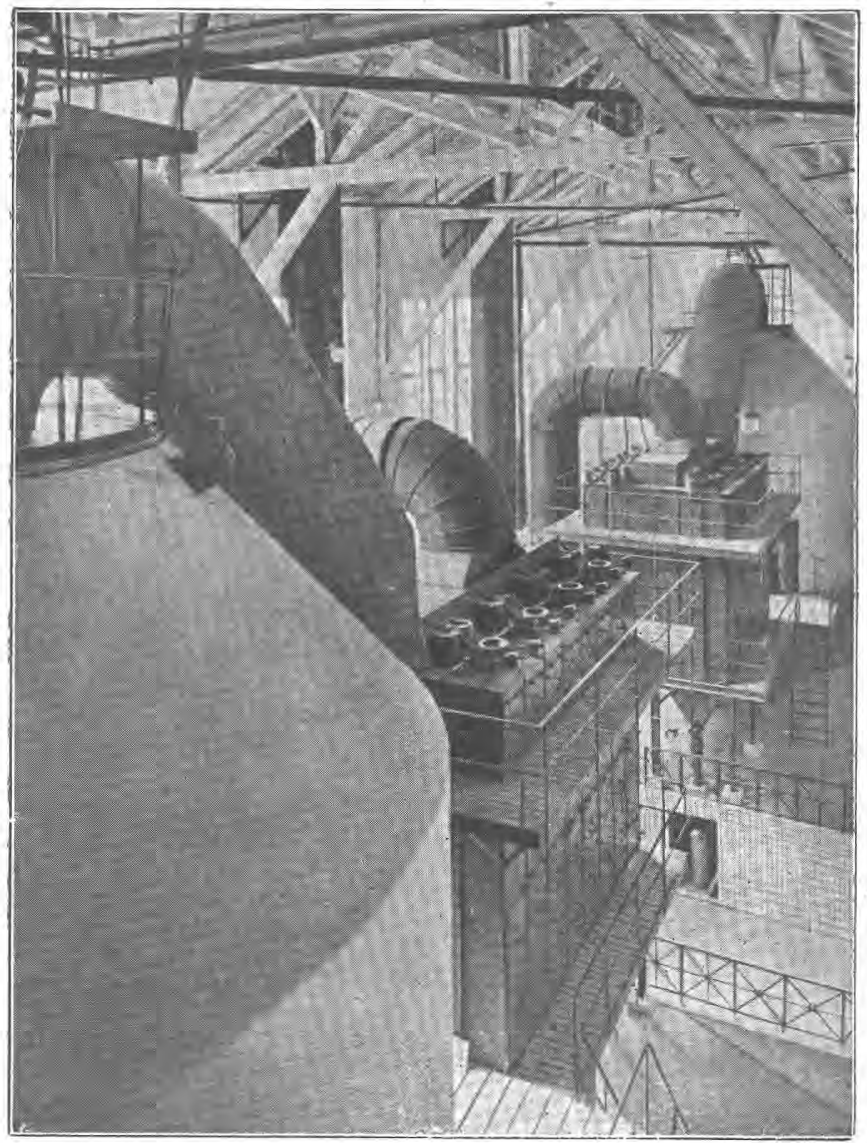

Tlall de dessiccalion pour deux appareils Krause, des Elablissements lailiers de l'Anglic, à Kappeln (Schleswig). (Vu de haut). 


\section{Figure II.}

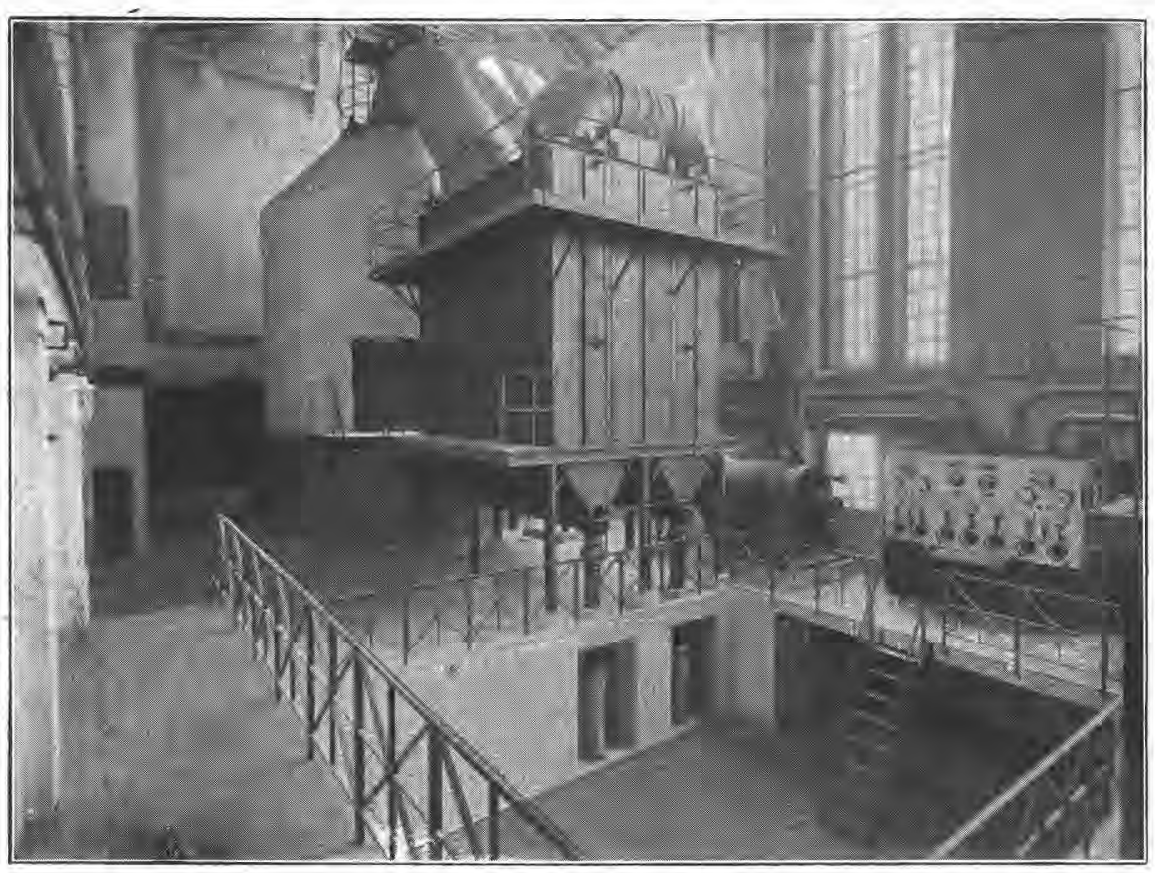

L'un des deux apparcils vu completement. Ne gauche a droite: la " tour », la chambre contenant deux séries de tuyaux filtes, chaque série déversant sa pondre sur une vis sans fin. A droite le turbo-ventilateur et la lable de manouvre commone aux deux apparcils. 
Figure III.

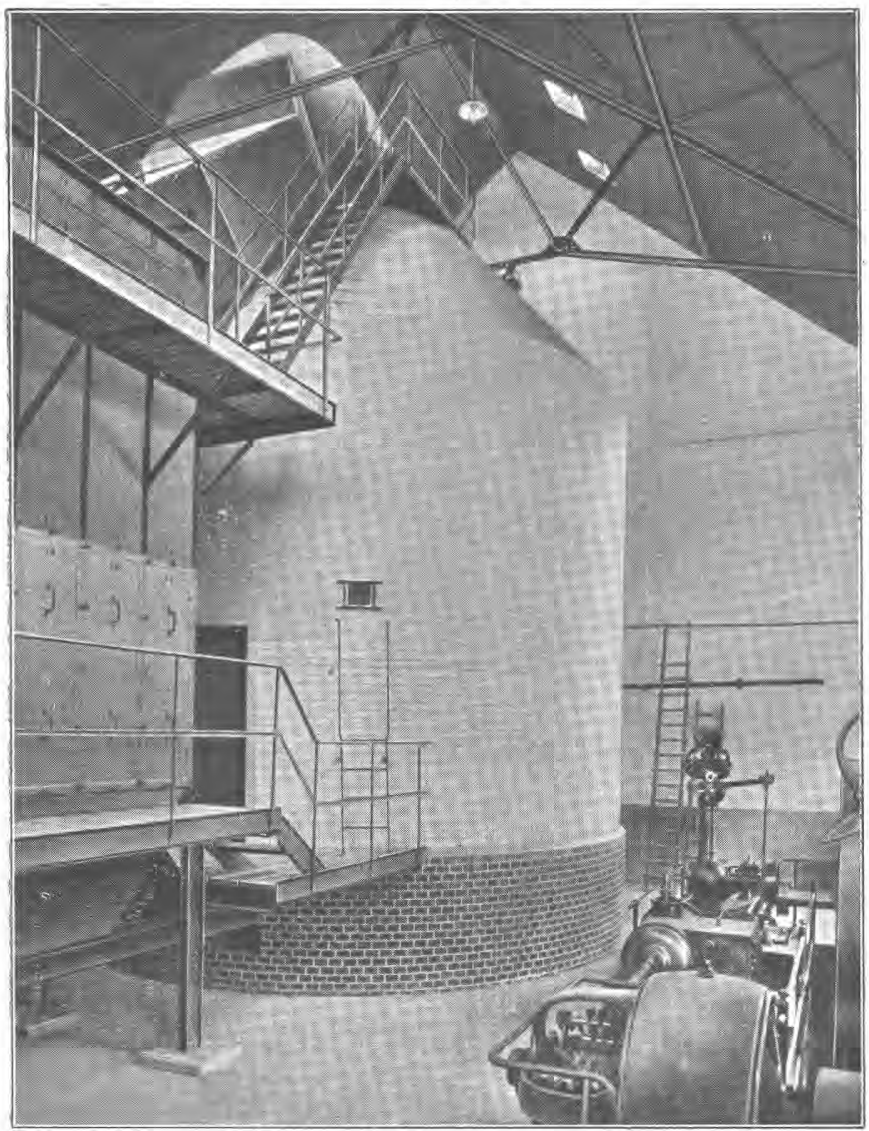

Inslallation pour la dessiccalion du lait et de la caséine à Gronau. A ppareil évaporant 1.003 litres d'eau à l'heure. Remarquer la porte donnant accès dans la tour el la fenctre permclland d'observer l'intérieur de la tour. 
Figure IV.

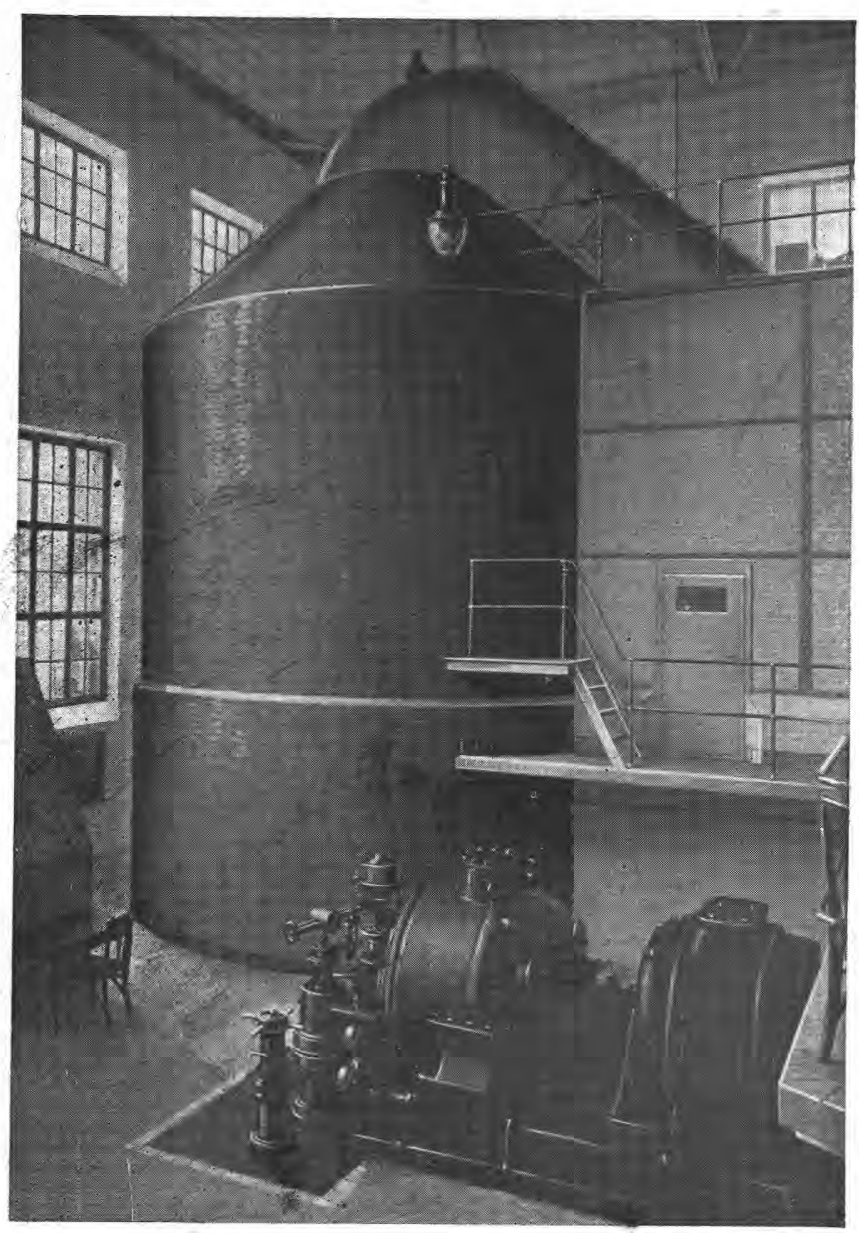

Un des deux appareils des Etablissements laitiers à Schlachter (Bavière). Type 1000 litres d'eau à l'heure. 


\section{Figure V.}

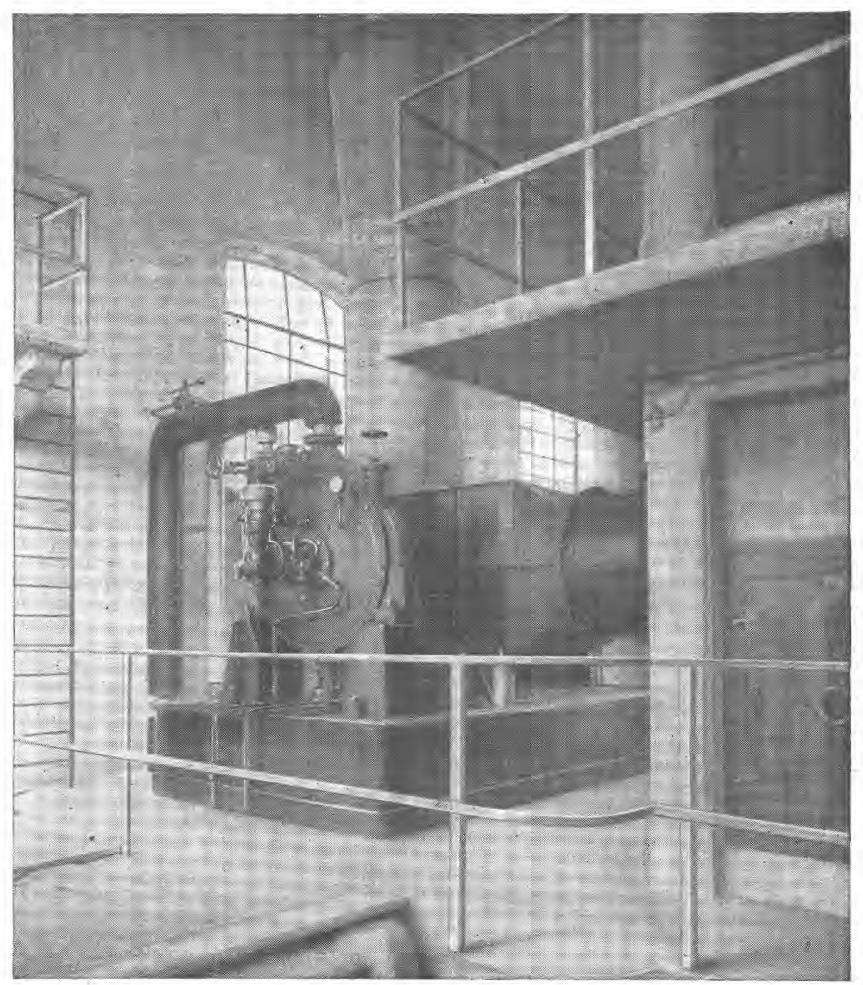

Turbo-ventilateur de l'installation Krause, pour la dessiccation du lait à Lodi (Italie). Type le plus moderne. 

est faible à basse température, et quedu fait de la rapidité de l'évaporation dans la tour, le degré de saturation de l'air doit être maintenu. assez bas. L'air chaud $\left(120^{\circ}-130^{\circ}\right)$ doit être dans la tour, refroidi à une température moyenne de $60^{\circ}$, variable avec le genre de fabrication. Dans ces conditions, il faudrait $2 \mathrm{~kg}$. de vapeur d'eau pour évaporer un litre d'eau, pratiquement, $2 \mathrm{~kg} ., 500$ pour un appareil type. 1000 litres a l'heure. Par suite d'améliorations, l'utilisation de la vapeur, pour le service de la turbine et du ventilateur a été réduite de telle façon que la vapeúr résiduaire est utilisée intégralement pour le chauffage de l'air. Lorsque la dessiceation est précédée d'une concentration du liquide dans le vide, on peut abaisser à un kilog le besoin en vapeur pour évaporer un litre d'eau de la substance à dessécher.

La conduite de l'appareil est très simple, il suffit d'un seul homme, même pour les appareils à fort débit.

Les brevets Krause sont exploités par la Metallbank und Metallurgische Gesellschaft A. G., à Francfort sur le Main.

\section{BIBLIOGRAPHIE.}

E. Philippe, Das Krause. - Trocknungsverfahren und seine Anwendung (Arbeiten aus dem Gebiele des Lebensmittel, 1919, 195). Cet article est le plus complet qui ait été écrit au point de vue scientifique et général sur le procédé Krause. Avec l'autorisation de M. Prilippe, j'en ai fait de très larges extraits.

Siegens, - Uber das Krauseverfahren. Zeitschrift fur angewandte Chemie, 1922 , p. 533.

Grimmer. - Article Mruch in Enzyklopaedie der technischen Chemie von Ulmanin, vol. 8.

Freund, E. -- Herstellung und Verwendung von Trockenmilch, Berlin, 1918. Metallbaxk und Metallurgische Gesellschaft. - Krause Verfahren.

Von Noorden. - Therapeutische Halbmonatshefte, vol. 35, 2 juillet 1921.

Neulann el Peipens. - Medizinische Klinik, no 28, 1921.

Czenny. - Fortschritte der Medizin, n²8, 14 déc. 1921 ; Le Lait, p. 456, 1921 ; p. 45, 1922.

Орек. - Blutgewinnung. Zeitschrift für Fleisch-u. Milchlıygiene, 1919, p. 141. Ströse. - Uber das nach dem Krause Verfahren getrocknete Blut. Zeits. für Fleisch-u. Milchhygiene, 1919, p. 61: 